Article

\title{
Colored Paints Containing NIR-Reflective Pigments Exposed to Accelerated Ultraviolet Radiation Aging with Possible Application as Roof Coatings
}

\author{
Stefano Rossi ${ }^{1, *(\mathbb{D})}$, Hampus Lindmark ${ }^{2}(\mathbb{D})$ and Michele Fedel ${ }^{1}$ (D) \\ 1 Department of Industrial Engineering, University of Trento, Via Sommarive 9, 38123 Trento, Italy; \\ michele.fedel@unitn.it \\ 2 Department of Chemistry and Chemical Engineering, Chalmers University of Technology, \\ Chalmersplatsen 4, 41296 Göteborg, Sweden; lhampus@student.chalmers.se \\ * Correspondence: stefano.rossi@unitn.it; Tel.: +39-0461-282442
}

Received: 28 August 2020; Accepted: 20 November 2020; Published: 23 November 2020

\begin{abstract}
This study aims to evaluate the difference in thermal behavior among paints with the presence of traditional and NIR pigments by means of a simple and cheap laboratory-scale test. Considering these goals, the thermal and esthetical properties of two different cool coatings were assessed, highlighting their positive and limited aspects. Two different complex near-infrared inorganic reflective (NIR) pigments with yellow and black respectably colors were mixed in an acrylic waterborne copolymer binder. The paint formulations were applied on steel panels. The thermal performance of the coatings was investigated in the NIR-region of the light spectrum by exposing the samples to an IR-lamp. The outer and inner surface temperatures of the painted panels were recorded using thermocouples and an IR camera. The samples were aged by artificial UV-B light exposure. Color and specular gloss changes at different exposure times were evaluated. The behavior of the cool coatings was compared with that of conventional coatings with similar color characteristics. The black cool coating achieved a maximum temperature decrease, compared to the conventional black one, of approximately $12{ }^{\circ} \mathrm{C}$. The stability for the cool coatings was very similar to that of the conventional coating, indicating that black pigment could be a potential candidate for cool-coating applications. The yellow cool coatings did not show a significant decrease in temperature compared to the conventional paint. The gloss and color changes resulted as influenced by the types and amount of pigments.
\end{abstract}

Keywords: complex NIR-reflective pigments; artificial UV-B radiation; cool coatings; thermal properties; color

\section{Introduction}

Following the global increase of population, the urbanization and expansion of cities increased with unprecedented speed throughout the last century [1]. Consequently, the modification of landmasses gave rise to the so-called Urban-heat island effect (UHI effect) [2,3]. Upon replacing natural material with building materials, an increase of absorbed irradiated solar light was observed in these regions, which caused a relevant rise in temperatures [3-6]. Literature studies reported that the UHI effect contributes to temperature increments that may exceed several degrees Celsius when compared to the adjacent rural environment [7]. As a direct consequence, an increase in usage of cooling devices, such as air conditioning, was observed in these areas, contributing to a rise of emission produced by fossil fuel use [8]. In addition, previous studies showed that the UHI effect might cause severe health risks in major cities that already experience high peak temperatures during summer due to their geographical location [9]. 
The sun radiation is commonly divided into several different regions depending on the energy of the electromagnetic wave. The VIS portion of light $(380-780 \mathrm{~nm})$ contains approximately $44 \%$ of the total solar energy. The optical property of a material in the VIS part of light determines its color characteristics. Nearly $50 \%$ of the total solar energy is radiated in the near-infrared (NIR) fraction of light $(780-2500 \mathrm{~nm})$ [10]. The optical properties in this region do not alter the color features of surfaces but have a strong impact on their thermal property [11,12].

The effect of buildings' presence on urban temperature has been exhaustively illustrated by Santamouris and coworkers [6,13-16], in particular in the Greek region [3,17]. Cool roofs and cool pavements could represent one of the most interesting solutions $[6,14,16,18]$ to tackle the UHI effect.

The rising energy costs and the increasing energy consumption observed due to the UHI effect, along with increased environmental awareness, have led to the development of new materials that lower the heat accumulation in buildings [19]. In this context, a cool roof in the form of ceiling tiles and other roofing materials were considered [13]. Painted steel and aluminum sheets are often used in roof construction in order to reduce building construction time and to save cost. The application of cool coating represents a readily studied approach to mitigate the UHI effect [6]. Compared to conventional coatings, this system exhibits a high reflection of incoming NIR light, which leads to a reduction of surface temperature compared to conventional coatings, without altering the color characteristics of the structure [20-22]. This result can be achieved by employing NIR reflective pigments in the coating. NIR pigments are colored pigments which do not have electronic transitions for wavelengths $>700 \mathrm{~nm}$ and, therefore, reflect the wavelengths in the infrared region. For this reason, the NIR reflective pigments containing coating represents a passive cooling system that does not require energy to operate.

Most of the studies devoted to cool roof materials investigate the VIS-IR reflective properties of the coatings using the spectrophotometer and the solar reflectometer $[10-13,19,20,23]$. This methodology is recognized to provide useful information on coating behavior. However, this approach is limited since it is not able to simulate a real system, which consists of a coating applied on a metallic substrate, as it does not consider the transmission of heat through the panel. In addition, the connection between the IR reflectivity of a surface and its actual tendency to heat up upon exposure to an IR light source is not straightforward.

The thermal behavior of cool coatings is generally assessed employing new samples without taking into account the possible influence of the deterioration of the coating due to aging of the polymeric matrix and/or of the pigments. As a matter of fact, the service life of cool coatings for exterior surfaces of buildings could be limited due to weathering effects [23]. An important factor that may influence the esthetical, mechanical and thermal properties of cool coatings is the degradation of the layer due to the absorption of incoming UV-B radiation (280-315 nm) [24]. Incoming UV-B light may be absorbed by the coating material, leading to photooxidative reactions, which could give rise to significant degradation of the binding material or pigments inside the coating. As a result, both the esthetic and mechanical features of the coatings are compromised. Furthermore, the alteration of the chemical characteristics of the coatings due to individuate if UV-B absorption may affect the thermal properties, as well. For roof coatings application, it is necessary to design cool coatings that achieve low susceptibility towards UV-B radiation. Therefore, it is important to assess the thermal and esthetical properties of the cool coating as a function of UV-B exposure in order to ensure the product achieves a satisfactory service life. Lastly, it is important to study an effective procedure to analyze these aspects in small lab-scale environments prior to large-scale, in order to estimate the coating's performance in a feasible and accessible way.

The purpose of this work is twofold. The first target is the development and validation of a simple and inexpensive test method to evaluate the thermal behavior of roof coatings. The second aim of the work deals with the assessment of whether the influence of aging can lead to significant changes in the thermal behavior of the roof coatings. In fact, outdoor exposure could promote a decrease in the efficiency of the roof coating, thus affecting its service life. 
The innovative aspect of this work is the development of a very easy and economical lab-scale method for assessing variation of thermal efficiency and esthetical parameters of cool coatings as a function of exposure to artificial UV-B radiation. A previous study $[25,26]$ aimed to assess the potential of this simple methodology as a reliable alternative to the most used tests $[10-13,19,20,23]$.

With the limit of the house simulacrum, the use of the small house in this work appears to be a simple method that provides useful data and allows to discriminate the thermal properties of the different paints. The data collection involves a simple contact temperature gauge of very limited cost, a house simulacrum and an IR lamp, typically employed in the field of gardening or breeding of small animals, with a cost of a few tens of euros. Regarding the geometry of the samples, the final use of the paint or the geometry of the building, it is also possible to easily modify the simulacrum geometry with very limited costs. Including the building materials for the simulacrum, the temperature sensors and the data recording system, the total cost was less than 700 euros. As a comparison, the cost of a reflectometer is higher than 60,000 euros. In a previous work [25], a simulacrum with a flat roof was used to make the experimental data collection easier. However, a simulacrum with the painted panels placed at a certain angle more realistically simulates the roof of most buildings. For this reason, in the present work, the house simulacrum geometry was modified. Despite the totally isolated house, without thermal transfer between walls, windows and doors, does not represent a real system, the studied setup allows to exclude external interference on the value of the measured temperatures. This perhaps represents one of the limits of this experimental approach, which, however, enables the evaluation of the behavior of the sample without external influences. A possible implementation of the system could be the execution of windows, simulating vents of the lab-scale model. However, in this case, the system would become more complicated as the transmission of heat through these openings should be considered. Another possible critical aspect is the fact that the measurements are carried out indoor, at room temperature: this represents a very different situation compared to the behavior of a building that is susceptible to the great variability of the ambient temperature. However, the system can be easily moved outside a building for outdoor measurements.

To validate the test methodology in the first work [25] industrially produced painted panels with fixed pigments quantity were used. Thus, a complete evaluation of the NIR reflectance efficiency of the pigments and the identification of their effective concentration was not possible.

In this study, each of two different NIR reflective pigments was mixed together with a polyacrylic binding material to obtain cool paints. The NIR reflective inorganic pigment amount for both cool coatings was varied between 1, 3 and $5 \mathrm{wt}$. $\%$ to determine how the pigment concentration affects the thermal features of the system.

Small model houses with roof panels coated with cool paint formulations were subjected to artificial NIR-light. The variation of thermal efficiency, gloss, color and coating degradation were analyzed as a function of time exposed to artificial UV-B light. UV-A and UV-B radiation exposition is a common method to simulate the natural weathering of organic coatings $[13,21,27]$. Possible critical aspects could be encountered. The UV component of solar radiation is one of the elements which contributes to the natural weathering of paints. In fact, UV-B and UV-A radiations are only portions of the solar spectrum. Moreover, humidity and other factors can influence the degradation of organic coatings and polymeric materials. Furthermore, no correlation was found between exposure to UV radiation and natural weathering. This aspect is also connected to the variation of natural degradation from area-to-area and from period to period. Therefore, natural degradation cannot be finely simulated, except by exposing the materials at the worksite. However, outdoor exposure would entail very long times, of many years, and it would provide results related only to the precise location of the exposure. The laboratory accelerated UV-B, and UV-A radiation exposure represents a well-established and widely used procedure to evaluate the degradation of polymeric materials, as demonstrated by a large number of references present in the literature [28-42]. Among the different accelerated tests, this work employed a UV-B radiation cabinet, which is able to promote the photochemical degradation of the coating (matrix and pigments). UV-B radiation is recognized to strongly affect the durability of organic 
coatings in relatively short times. UV-B-humidity cycles were not considered because the adhesion between coatings and the substrate is not the aim of this work; in addition, the possible formation of blisters and water accumulations could interfere with the interpretation of the thermal behavior data. Following ASTM G154 [43], $480 \mathrm{~h}$ of exposure were carried out, as considered to mimic the natural changes in pigmented paints. Clearly, other material durability tests require much longer exposure time [13]. However, for the aim of this research, this time could be considered sufficient to get the first information about color and gloss changes.

\section{Materials and Methods}

\subsection{Materials}

In this study, commercially available NIR reflective inorganic pigments were considered. These pigments do not absorb light in the near-infrared region. Thanks to their specific composition, energy transitions do not occur when exposed to near-infrared light (wavelength $>700 \mathrm{~nm}$ ). Thus, the NIR wavelengths are not absorbed but refracted, reflected and scattered, leading to diffuse reflection of NIR light [44]. The complex NIR reflective inorganic pigments used in this study were: (1) a black pigment based on chrome iron hematite (PS 24-10466, supplied by Ferro Company, Almassora, Spain) and (2) a yellow pigment based on a chrome antimony titanium buff rutile structure (PS 10406, supplied by Ferro Company, Almassora, Spain). The composition and the structural features of the NIR reflective pigments declared by the supplier were checked by $\mathrm{X}$-ray diffraction analyses (XRD). The black pigment exhibited the characteristic peaks of two phases: $\mathrm{Cr}_{1.3} \mathrm{Fe}_{0.7} \mathrm{O}_{3}$ in spinal structure and $\mathrm{Cr}_{2} \mathrm{O}_{3}$ in eskolaite structure. The yellow pigment consisted of a two-phases system containing a high amount of pure $\mathrm{TiO}_{2}$ in the rutile phase and a smaller concentration of $(\mathrm{Ti}, \mathrm{Cr}, \mathrm{Sb}) \mathrm{O}_{2}$ with an unclear structural phase. The XRD data were found to be consistent with the data reported in the technical datasheets of the commercial pigments provided by the supplier [45]. According to the composition of the investigated pigments, the literature reports that: (1) the chrome iron hematite structure provides a NIR reflectance over white of about $0.23-0.40$ when incorporated in an organic coating; while (2) the chrome antimony titanium buff rutile provides a NIR reflectance over white of about $0.80-0.86$ when incorporated in an organic coating [46].

The morphology and color of the pigments are shown in Figure 1. The scanning electron microscopy (SEM) images (secondary electron detector, SE) were obtained from an SEM (JEOL IT 300, Akishima, Tokyo, Japan).

The acrylic copolymer used as binding material was a pigment-free transparent paint (Aqualack, Alpina Italia, Vermezzo, MI, Italy). The FT-IR spectrum of the binder (free of any pigment inside) was collected to confirm the chemistry claimed by the supplier (it is uploaded with the correlation of characteristic peaks as additional material). The colored standard acrylic copolymer paints (which contain conventional, not NIR reflective pigments) used for comparison purposes in this project were purchased from MaxMeyer (Porcari, LU, Italy): NCS-S 2070Y20R yellow paint and NCS-S 8500 N black paint [47].

An acrylic clearcoat was selected due to the good weathering resistance of such a kind of resin. A pigment-free paint was employed in order to better highlight the effect of the sole NIR pigment on the thermal properties of the coatings. For comparison purposes with the NIR pigmented samples, a commercial conventional pigmented acrylic paint based with comparable RAL was employed.

The coatings were applied on steel panels $(152 \mathrm{~mm} \times 75 \mathrm{~mm} \times 0.1 \mathrm{~mm})$ purchased from Q-Lab (Westlake, OH, USA). A conventional white paint containing $\mathrm{TiO}_{2}$ (MaxMeyer, Porcari, LU, Italy) was applied as a primer on the metal substrate prior to top coating application. 

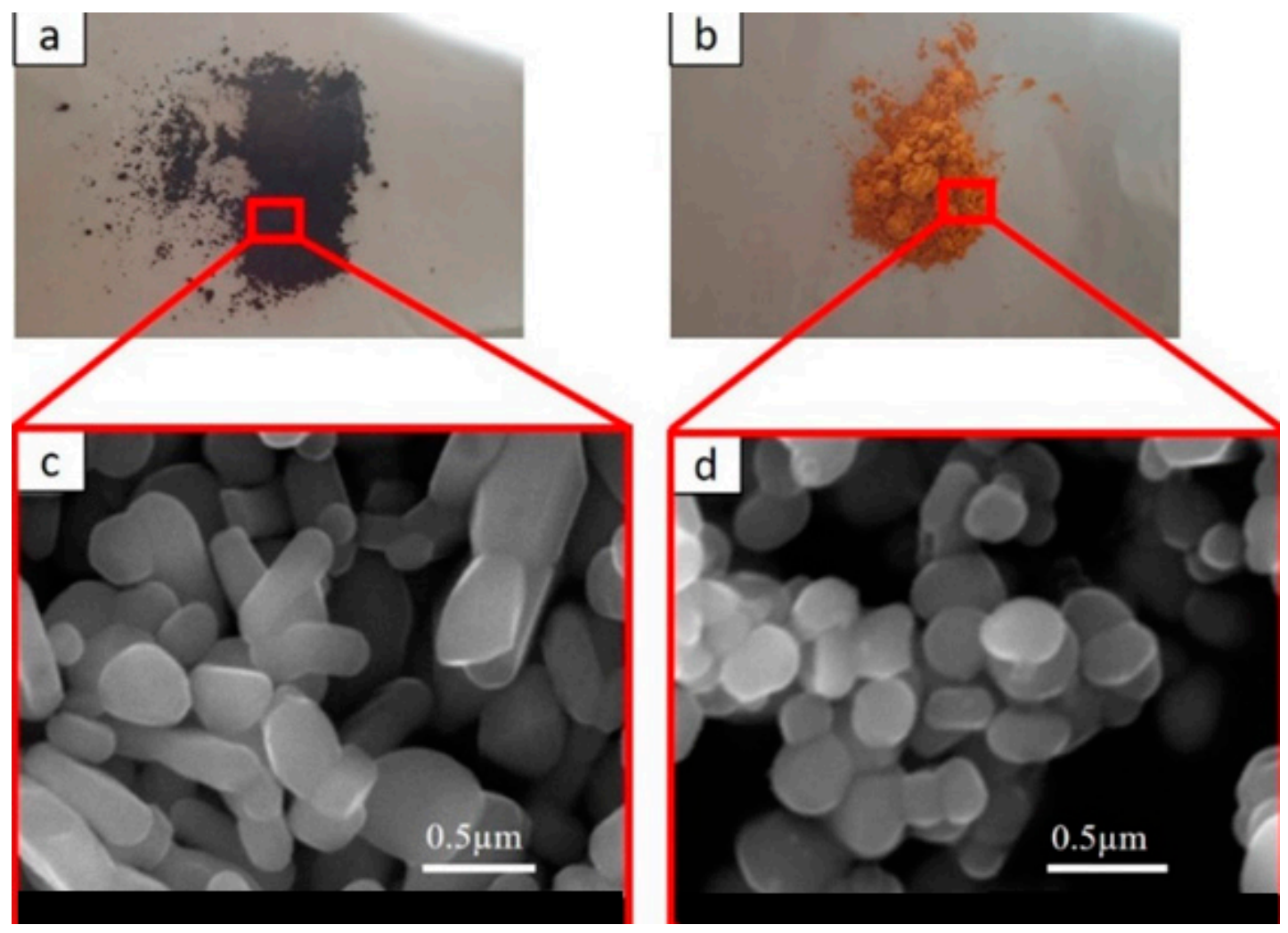

Figure 1. (a) Black near-infrared inorganic reflective (NIR)-reflective pigment; (b) yellow NIR-reflective pigment. SEM images of (c) black NIR-reflective pigment (d) yellow NIR-reflective pigment.

\subsection{Paint Formulation}

Initially, 1, 3 and $5 \mathrm{wt} . \%$ of each NIR-reflective pigment type were individually mixed in a beaker containing the transparent acrylic paint. The mixture was stirred in a motor-driven blade mixer (IKA ${ }^{\circledR}$ RW 20 digital, Staufen, Germany) for $1 \mathrm{~h}$ with a rotational speed of 550 rounds per minute (rpm). Subsequently, the paints were ultrasonicated (Hielscher ultrasound technology UP400S, Teltow, Germany) for 10 min using 70\% amplitude and one cycle mode. During the mixture step, a large amount of unwanted air was admixed into the paint, resulting in the possible formation of air bubbles. To prevent the risk of air trapping inside the coatings, the paints were sealed and stored at room temperature for $12 \mathrm{~h}$. Prior to coatings application, the metal substrates were sandblasted and cleaned with acetone and demineralized water.

\subsection{Paint Application}

A schematic representation of the application of the developed paints on the steel substrate is shown in Figure 2. The paints were sprayed on the treated metal substrates using a spray gun and $\mathrm{N}_{2}$ as pressure gas. The white primer paint was initially spray-deposited on the metal substrate and dried at room temperature for $24 \mathrm{~h}$. The presence of the white primer of equal thickness for all the samples allows discriminating the behavior of the top layers, considering the type and quantity of pigments.

After the base paint coating had dried, the formed cool and conventional paints were sprayed on top of the base paint and dried at room temperature for $8 \mathrm{~h}$. The coated samples were then stored at room temperature. A thickness greater than 30 microns of the top layer was chosen to completely cover the primer due to the lower covering power of this formulation.

The thicknesses of the primer and top coatings, summarized in Table 1, were measured by means of a digital thickness gauge (Phynix Surfix, Neuss, Germany). Comparing the thickness of the samples, the coatings with $1 \%$ exhibited higher values. 


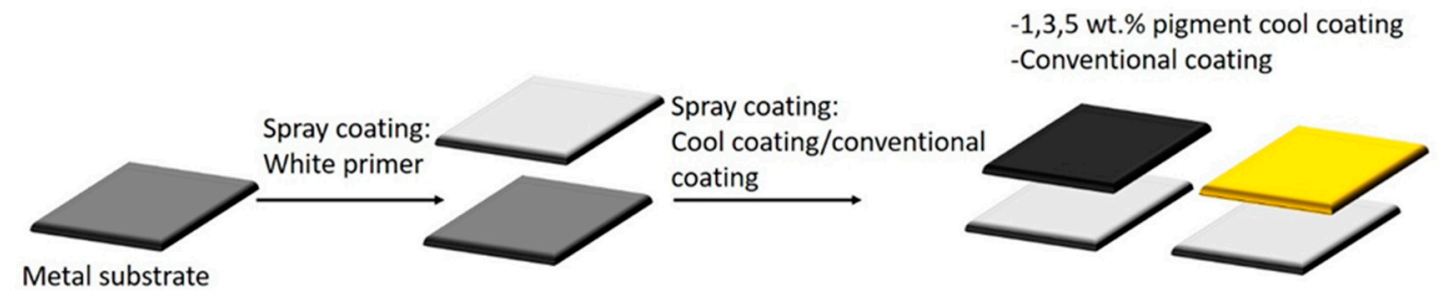

Figure 2. Paint application.

Table 1. Dry film thickness of each coating.

\begin{tabular}{cccc}
\hline Top Coating Color & Sample & Top Coating $(\mu \mathrm{m})$ & White Primer $(\mu \mathrm{m})$ \\
\hline \multirow{3}{*}{ Yellow } & 1 wt.\% cool coating & $54.0 \pm 12.3$ & $29.4 \pm 3.1$ \\
& 3 wt.\% cool coating & $39.5 \pm 3.4$ & $25.2 \pm 1.7$ \\
& 5 wt.\% cool coating & $38.3 \pm 6.4$ & $35.3 \pm 4.6$ \\
& Conventional coating & $37.4 \pm 2.2$ & $25.3 \pm 1.7$ \\
\hline \multirow{3}{*}{ Black } & 1 wt.\% cool coating & $69.7 \pm 13.0$ & $38.4 \pm 8.7$ \\
& 3 wt.\% cool coating & $37.1 \pm 5.3$ & $27.7 \pm 2.9$ \\
& 5 wt. $\%$ cool coating & $32.8 \pm 6.6$ & $25.5 \pm 3.4$ \\
& Conventional coating & $31.0 \pm 4.3$ & $23.2 \pm 6.0$ \\
\hline
\end{tabular}

Ten data points were collected for each sample, and the recorded results were represented as the arithmetic mean of the obtained data points. The dispersion of the data was attributed to the deposition technique (spray coating), which does not allow optimal control of the amount of paint deposited on the substrate.

\section{Characterization Techniques}

\subsection{Gloss and Color Samples Analysis}

The gloss for each coating was measured using a Picogloss model 503 Erichsen Glossmeter instrument (Hemer, Germany) at a $60^{\circ}$ angle. Five data points were collected for each sample in different areas of the exposed surface to have representative data of the whole coating area. The recorded gloss values were represented as the arithmetic mean of the obtained data points. The color measurements were conducted using a CM-2600d spectrophotometer (Konica Minolta, Chiyoda-ku, Tokyo, Japan) with an observer angle of $10^{\circ}$ and an opening diameter of $10 \mathrm{~mm}$. These measurements were carried out in accordance with CIEL $a^{*} b^{*}$ color space method $[48,49]$. The CIEL* $a^{*} b^{*}$ color space method is an approximated system where all apparent colors visible to the human eye are described mathematically in a 3D color coordination system. The axes represent three different color characteristics $\left(L^{*}, a^{*}\right.$ and $\left.b^{*}\right)$. The L-axis corresponds to the brightness of the paint, $L^{*}=100$ represents the white color, and $L^{*}=0$ correlates to a totally dark color sensation. The $a^{*}$ and $b^{*}$ chromatic axes represent color opponent dimensions. The positive a-axis goes from a negative value that corresponds to green color towards a positive value that is red. Positive $b^{*}$ value represents yellow color, while negative $b^{*}$ value stands for blue color. The color change of each sample after exposure using the three different coordinates can be described with one single value $(\Delta E)$, mathematically expressed as follows:

$$
\Delta E=\left(\Delta a^{* 2}+\Delta b^{* 2}+\Delta L^{* 2}\right)^{1 / 2}
$$

where $\Delta a^{*}, \Delta b^{*}, \Delta L^{*}=a^{*}, b^{*}, L^{*}$ initial $-a^{*}, b^{*}, L^{*}$ post-exposure. Five measurements were carried out on the whole surface of the sample in order to have representative data of the whole exposed surface and not just limited to a small selected area. 


\subsection{Thermal Behavior Evaluation}

The setup for the thermal evaluation of the coatings is illustrated in Figure 3c.

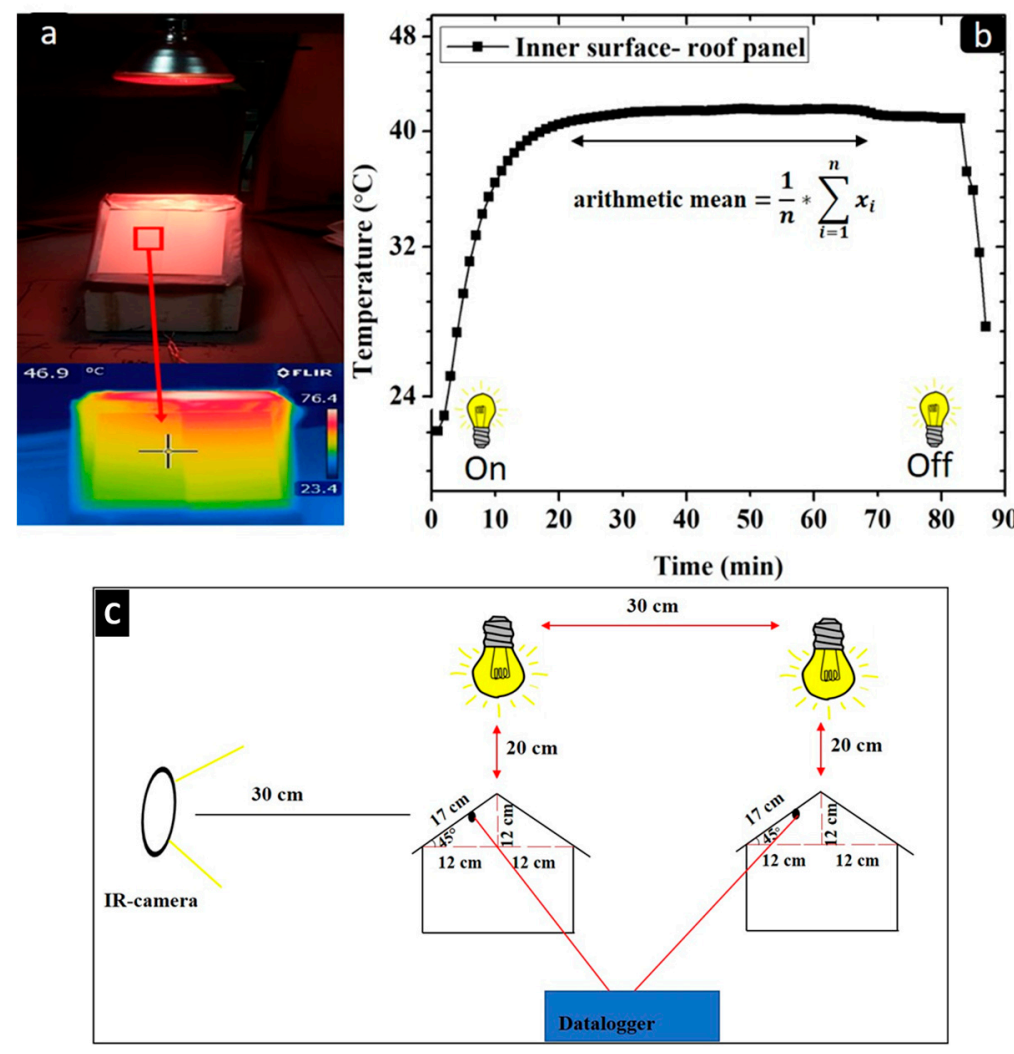

Figure 3. (a) IR-image obtained by an IR camera of the outer surface of the roof panel; (b) temperature profile of the inner surface of roof panel during measurement; (c) experimental setup for the thermal measurements of the coated metal substrates.

The coated samples were applied as roof panels on a small-scale house model with a roof angle of $45^{\circ}$ (2 panels for each part of the roof). Each sample was exposed under an IR lamp (Philips BR125 IR $150 \mathrm{~W}$, Philips, Eindhoven, The Netherlands) that was placed $20 \mathrm{~cm}$ above the roof panels.

The lamp irradiation used does not correspond exactly to the solar spectrum, as the lamps are focused on IR radiation. They were chosen to evaluate the thermal behavior of the different coatings containing NIR pigments and to highlight the differences between the samples.

Thermocouples were applied on the rear part of the roof panel substrate. The thermocouples were connected to a Delta OHM HD 32.7 RTD data-logging instrument (Delta Ohm, Caselle di Selvazzano, PD, Italy) that recorded and collected temperature data every $60 \mathrm{~s}$. The experimental procedure continued until the inner surface temperature of the roof panel reached a stable temperature plateau. The inner roof panel temperature recorded by the thermocouple was represented as the arithmetic mean temperature of the observed temperature plateau (Figure 3b).

The outer surface temperature of the coatings was recorded using an IR camera (FLIR-T62101, Flir, Santa Barbara, CA, USA). The IR images were taken at $30 \mathrm{~cm}$ in front of the house model setup and approximately at the same position as the thermocouple, recording the internal temperature of the metal roof panel. Several IR images for each sample were taken, and the recorded result was represented as an average (arithmetic mean) value obtained from the recorded images (Figure 3a). The IR images were taken at the end of each thermal measurements, and the emittance of each coating sample was estimated to be equal to 0.9. The thermal measurements were collected every $96 \mathrm{~h}$ of UV-B exposure for a total of $480 \mathrm{~h}$. 


\subsection{Lab Scale Weathering}

The painted samples were exposed in a laboratory UV-B cabinet (UV-Chamber, Erichsen, Hemer, Germany) to highlight the possible influence of coatings weathering damage on their thermal behavior.

The accelerated UV-B test was carried out in compliance with the ASTM-G154-06 standard using UV-B 312-EL Hg lamps as the light source. The total exposition time was $480 \mathrm{~h}$ because it proved to be sufficient to provide a relevant color change of the investigated materials and to point out the difference among the coatings.

Every $96 \mathrm{~h}$ of UV-B exposure, the thermal behavior and the changes of gloss and color (also affected by the different nature and percentage of the pigment in the different coatings) were checked to investigate the potential correlations among these parameters. Three data points were collected for each evaluation position, for a total of 15 measurements per sample.

The chemical degradation of the coatings as a function of accelerated UV-B exposure was assessed using FT-IR spectroscopy in attenuated total reflection (ATR) mode. The analysis was carried out using a Varian 4100 FT-IR Excalibur series instrument (Santa Clara, CA, USA). A resolution of $1 \mathrm{~cm}^{-1}$ and a spectrum of wavelength ranging from $600-4000 \mathrm{~cm}^{-1}$ were used.

\section{Results and Discussion}

\subsection{Distribution of Pigments}

The dispersion of the pigments inside the acrylic coatings was assessed by means of SEM observation of the cross-sections of the samples. Figure 4 exhibits an almost homogeneous dispersion of both yellow and black pigments in the acrylic paint. However, a partial agglomeration of the pigments seemed to occur since 1-2 $\mu \mathrm{m}$ particles were detected.

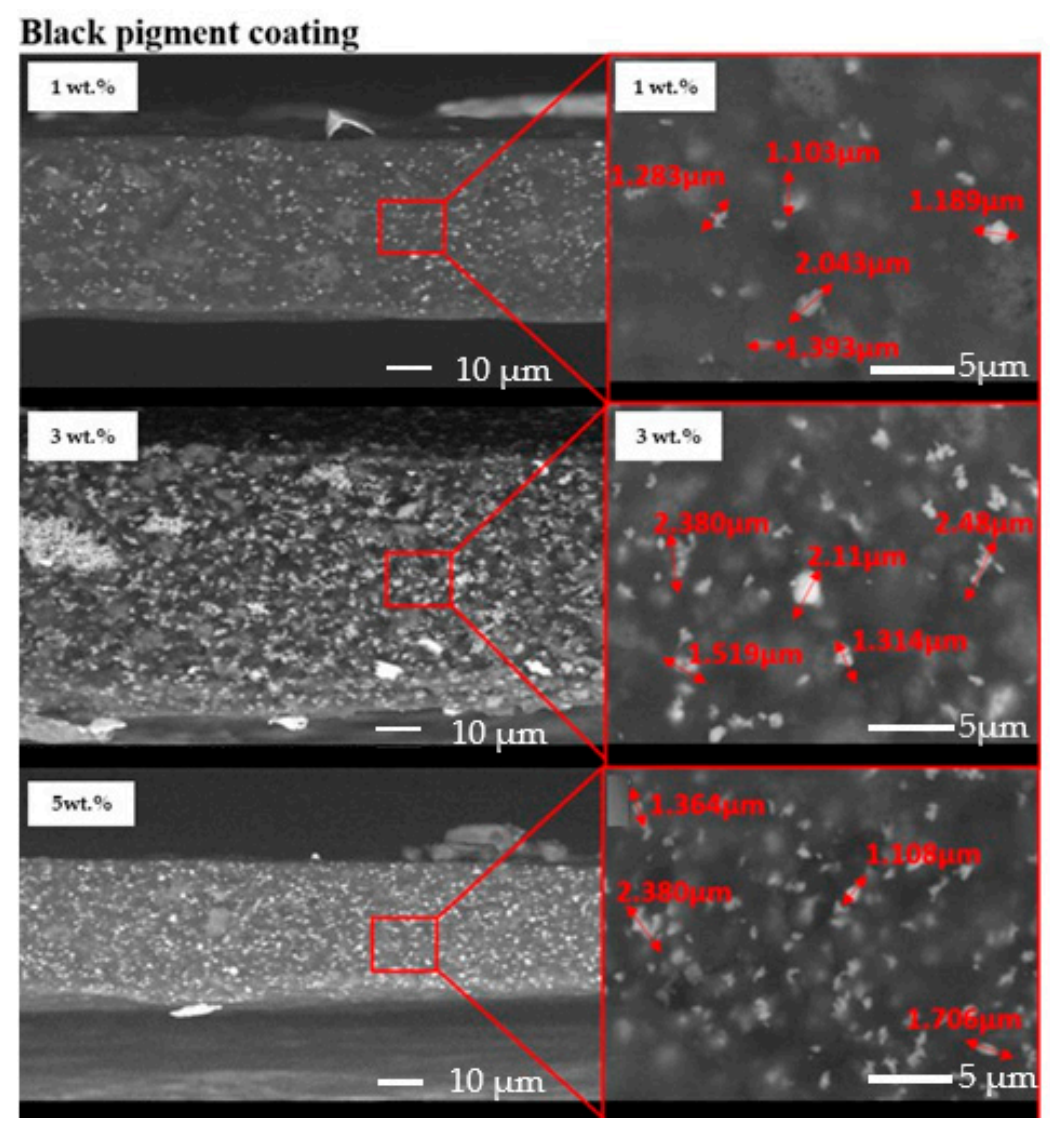

(a) 


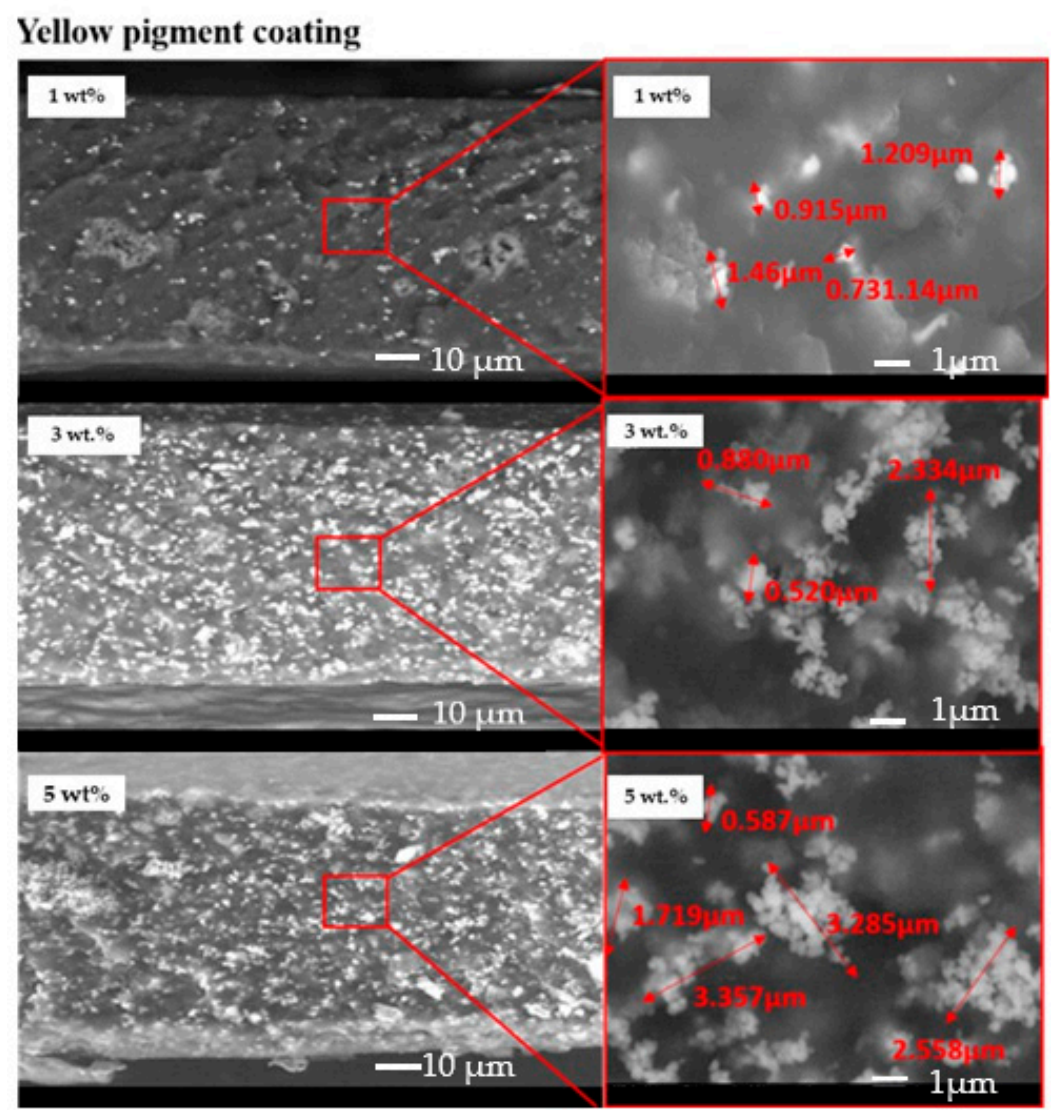

(b)

Figure 4. Cross-sections of the investigated samples: black pigmented coatings (a) and yellowpigmented coatings $(\mathbf{b})$.

\subsection{Thermal Evaluation}

Figure 5 shows the achieved inner and outer temperatures for each coated metal sample as a function of time exposed to UV-B radiation.

First, in all cases, the outer surface temperature result was higher than the inner one. This aspect was connected with the heat diffusion through the two layers of organic coatings and the thickness of the substrate. The surface of the sample was irradiated by the lamp and heated. The heat was then transmitted by conduction to the rear part of the panel (outer surface $\rightarrow$ inner surface). Since the external surface was continuously irradiated and the diffusion of heat was not immediate, a temperature difference between the two external and internal surfaces was detected. In addition, as illustrated in Figure 3, the data were collected after the first stabilization of the temperature.

A significant reduction in outer and inner surface temperatures of the metal panels was observed for the black cool-coated samples compared to the black conventionally coated ones. The recorded temperature maximum difference was observed for the outer part of the $1 \mathrm{wt} . \%$ sample at the initial state and corresponded to a temperature difference of $12{ }^{\circ} \mathrm{C}$ compared to the conventional coating. After $480 \mathrm{~h}$ of UV-B exposure, the maximum temperature difference was approximately $10^{\circ} \mathrm{C}$ compared to the conventional one and was recorded for the $3 \mathrm{wt} . \%$ sample. This aspect may indicate lower effectiveness of the NIR pigments after UV exposure that may be partially shielded from the degradation of the matrix. In addition, no significant temperature deviation was detected as a function of pigment concentration. 


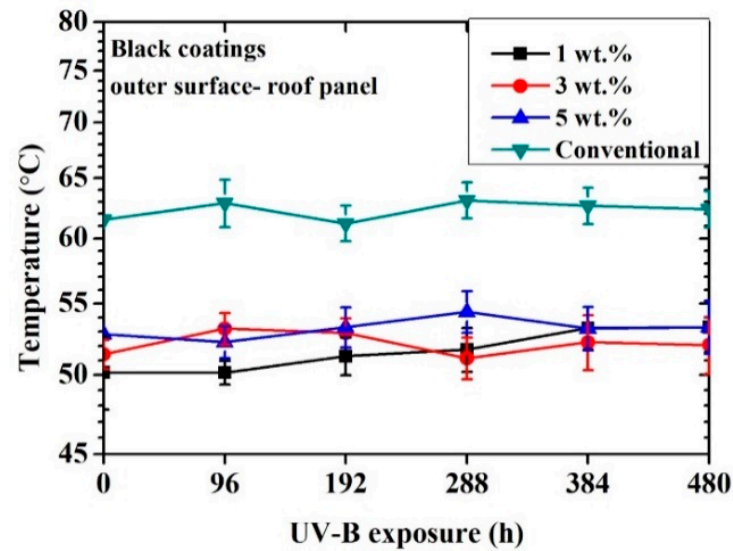

(a)

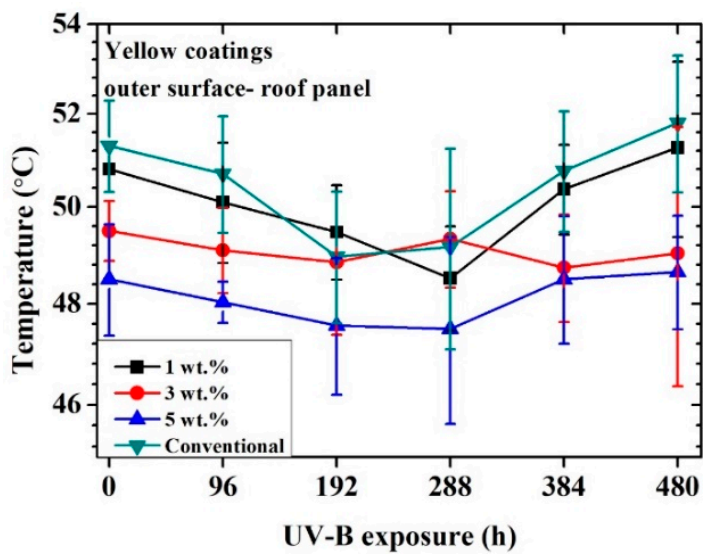

(c)

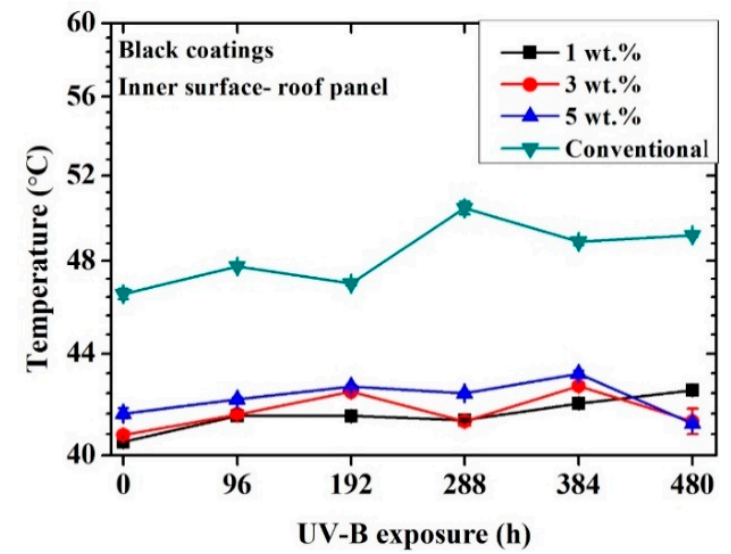

(b)

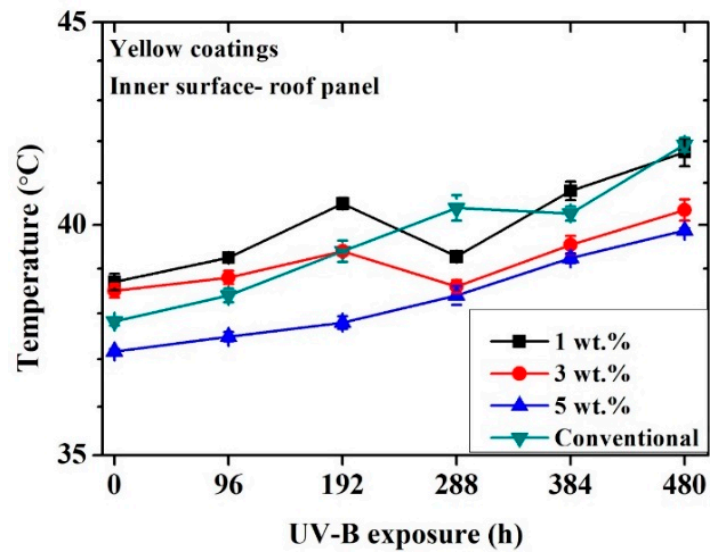

(d)

Figure 5. Temperature profile vs. time exposed to artificial UV-B light: black coatings outer surface of roof panel (a), black coatings inner surface of roof panel (b), yellow coatings outer surface of roof panel (c), yellow coatings inner surface of roof panel (d).

The temperature profile for each black coating, including the conventional one, did not significantly change after $480 \mathrm{~h}$ of UV-B exposure. Considering that the thermal analysis was conducted in the NIR section of light and that the conventional coatings achieved a similar color as the cool coatings, these results suggest that a significant reduction of absorbed light was achieved for the black cool coatings regardless of the pigment amount.

The yellow cool coatings reached similar temperatures as the conventional system. However, in observing the temperature profile, a limited decrease in temperature was exhibited as a function of pigment concentration. The highest difference between the conventional sample and the samples containing NIR pigments was about $2{ }^{\circ} \mathrm{C}$ for the sample with $5 \%$ pigments. This aspect implies that a higher pigment wt.\% was required for these samples to achieve a more significant temperature reduction. The temperature of the cool coatings remained similar even after $480 \mathrm{~h}$ of UV-B exposure.

The thickness of the coatings did not appear to had a significant influence. For example, considering the black samples, the $1 \%$ and $3 \%$ coatings showed similar thermal behavior (at time 0 , less than $1{ }^{\circ} \mathrm{C}$ of difference) regardless of the different thickness.

Figures 6 and 7 show the thermal measurements for the black and yellow $5 \mathrm{wt} . \%$ cool coatings, respectively and the conventional one. The IR images represent only one of many images that were taken for each data point to achieve arithmetic mean temperature value. The temperature plateau of the inner surface was achieved after approximately $25 \mathrm{~min}$, regardless of coating type or pigment load. Considering the not-weathered black samples $(0 \mathrm{~h})$, a noticeable temperature difference between NIR 
and the conventional coating was observed. This effect was attributed to the nature of the NIR pigment, which reflected a significant portion of the infrared light. The difference in the plateau temperature between the conventional and the black cool coatings increased after $480 \mathrm{~h}$ of exposure in the UV chamber. The poorer performance upon UV-B exposure of the conventional coating may be attributed to the surface modification of the coating in terms of gloss and color, as illustrated below. The presence of the NIR pigment seemed to prevail over the degradation of the polymeric matrix since the surface of the $5 \mathrm{wt} . \%$ black cool coating was also affected by the UV-B light. In fact, the $5 \mathrm{wt}$ \% black cool coating showed approximately the same inner temperature after $480 \mathrm{~h}$ of exposure in the UV cabinet, while the standard black coating decreased in IR reflectance after the same accelerated aging treatment.

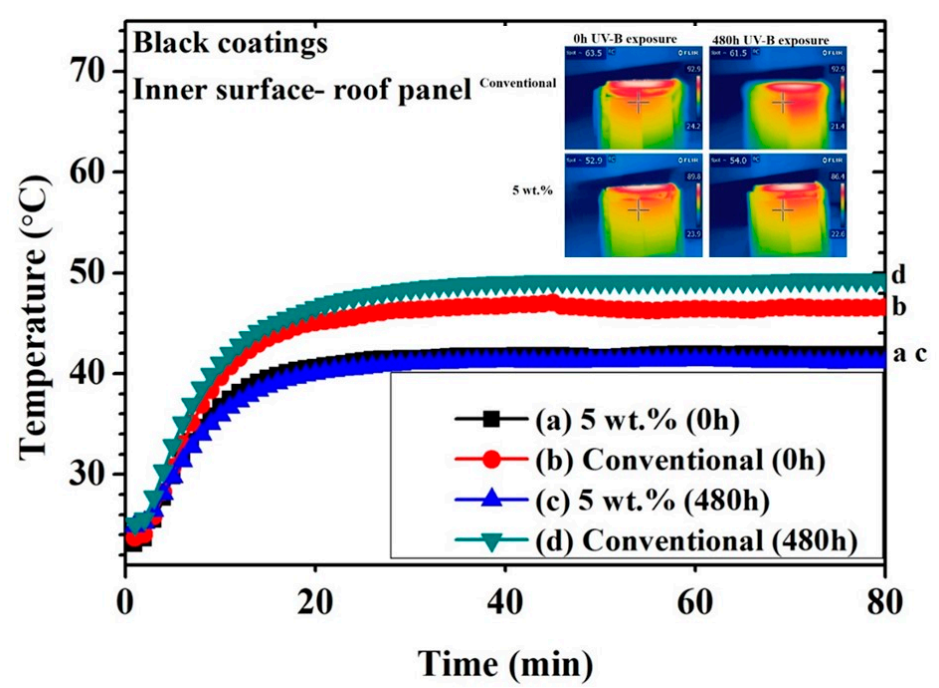

Figure 6. Thermal measuring points for black coatings (inner surface of roof panel) obtained with a thermocouple and an IR camera.

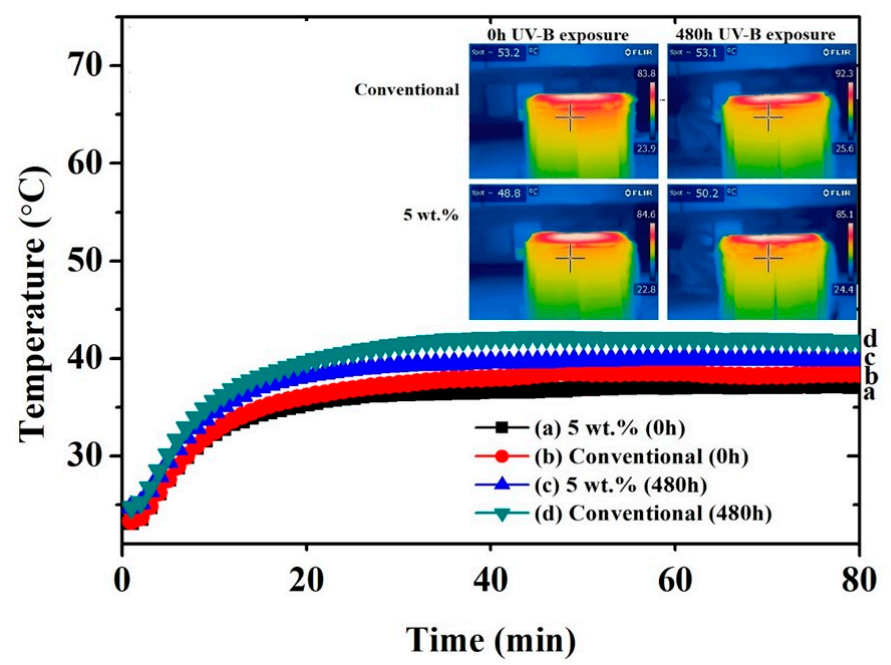

Figure 7. Thermal measuring points for yellow coatings (inner surface of roof panel) obtained with a thermocouple and an IR camera.

The difference between the conventional and the NIR pigment samples was less marked in the case of the yellow pigment. This behavior may have been due to the difference between black and yellow colors. The black color tended to increase its temperature more remarkably in comparison to the yellow one $[23,50,51]$, and therefore it was easier for the black NIR pigment to show its effectiveness. However, comparing the behavior of the yellow samples, it was also possible to observe a reduction of 
the temperature in the case of the coatings containing the NIR pigments, even if the effect was less marked. Again, the effect of the weathering on the temperature trend was noticeable.

\subsection{Gloss and Color Evaluation}

Table 2 summarizes the initial color for each coating sample represented as CIEL $L^{*} a^{*} b^{*}$ color coordinates.

Table 2. Initial color coordinates for each coating sample.

\begin{tabular}{ccccc}
\hline \multirow{2}{*}{ Color } & Sample & \multicolumn{3}{c}{ Color Coordinates } \\
\cline { 3 - 5 } & & $\boldsymbol{L}^{*}$ & $\boldsymbol{a}^{*}$ & $\boldsymbol{b}^{*}$ \\
\hline \multirow{3}{*}{ Yellow } & 1 wt.\% cool coating & $75.04 \pm 0.85$ & $14.01 \pm 0.44$ & $69.14 \pm 0.63$ \\
& 3 wt.\% cool coating & $70.77 \pm 0.13$ & $21.89 \pm 0.24$ & $75.59 \pm 0.23$ \\
& 5 wt.\% cool coating & $69.38 \pm 0.14$ & $24.71 \pm 0.03$ & $74.22 \pm 0.16$ \\
& Conventional coating & $72.96 \pm 0.07$ & $25.99 \pm 0.19$ & $81.73 \pm 0.32$ \\
\hline \multirow{3}{*}{ Black } & 1 wt.\% cool coating & $18.38 \pm 0.43$ & $4.65 \pm 0.44$ & $4.52 \pm 0.72$ \\
& 3 wt.\% cool coating & $11.50 \pm 0.13$ & $6.72 \pm 0.21$ & $5.84 \pm 0.17$ \\
& 5 wt.\% cool coating & $7.85 \pm 0.14$ & $8.69 \pm 0.34$ & $6.85 \pm 0.34$ \\
& Conventional coating & $6.50 \pm 0.0$ & $6.29 \pm 0.0$ & $4.86 \pm 0.10$ \\
\hline
\end{tabular}

The samples containing $1 \mathrm{wt} . \%$ reflective pigment achieved a slightly lighter color sensation compared to higher pigment-loaded samples, regardless of the pigment type. The color difference could be easily detected by the naked eye inspection (the corresponding figure was reported as additional material).

Figure 8a shows the evolution of gloss measured for each coating as a function of UV-B exposure time. A very small alteration in gloss was observed for each cool coating, indicating a very low susceptibility of the coatings towards UV-B radiation. The initial gloss of each coating results decreased as a function of pigment load.

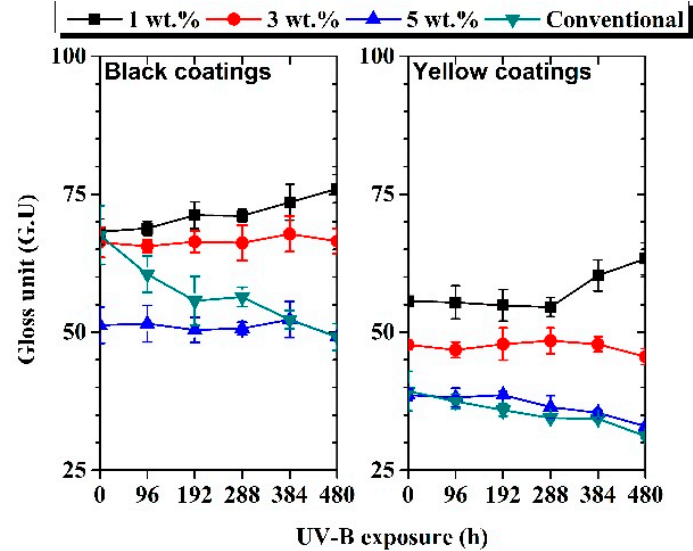

(a)

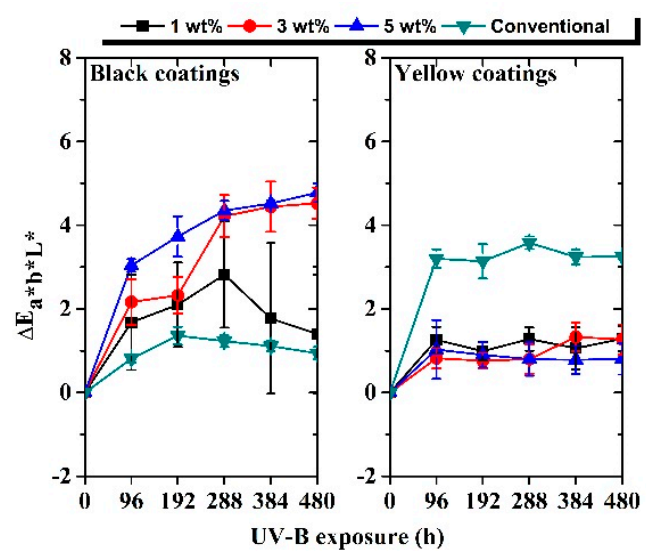

(b)

Figure 8. Evolution of $60^{\circ}$ gloss units (a) and color variation, $\Delta E_{L^{*} a^{*} b^{*}}(\mathbf{b})$, as a function of UV-B exposure time.

Compared to conventional coatings, the cool coatings containing pigments exhibit higher gloss stability. The gloss values remain almost constant or show a slight increase. This change could be connected with the modification of the layer surface introduced by UV-B exposure. Figure 9 shows the SEM pictures (BED-T, backscattering topographic modality) of the $3 \mathrm{wt} . \%$ black sample (a) before and (b) after $96 \mathrm{~h}$ of UV-B exposure. 


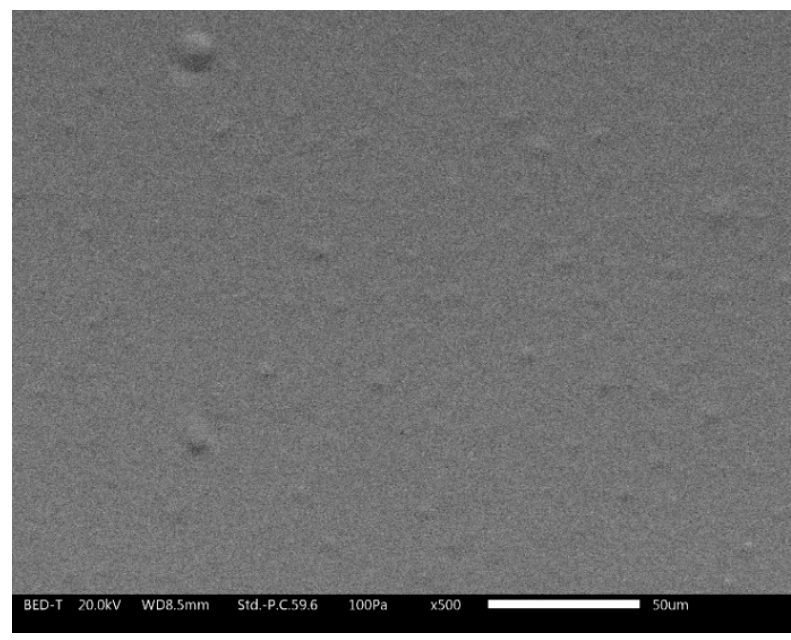

(a)

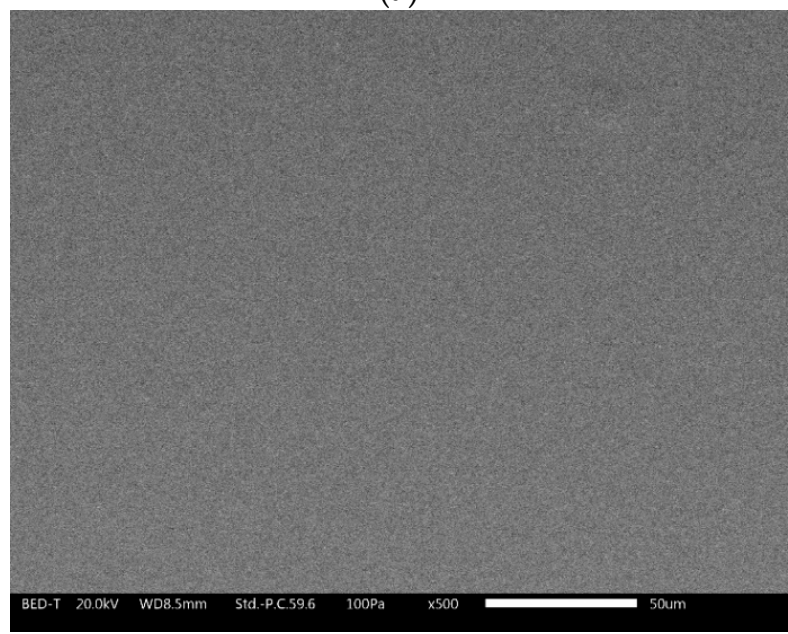

(b)

Figure 9. SEM (BED-topographic) pictures of the sample $3 \mathrm{wt} . \%$ at time 0 (a) and after $96 \mathrm{~h}$ of exposure (b).

Before the UV-B exposure, the sample showed a rougher surface with the presence of bumps. After $96 \mathrm{~h}$ of UV-B exposure, the surface seemed to flatten. The exposure to UV radiation locally increased the surface temperature and may have favored the evaporation of possible residual solvent; moreover, if the local temperature exceeded the $\mathrm{Tg}$ of the polymer, it was possible to also have distension of the material. This surface appearance was also maintained under prolonged UV-B exposure.

The color evolution, represented as $C I E L^{*} a^{*} b^{*}$ color coordinates, observed for each coating as a function of UV-B exposure time, is represented in Figure 8b. For each cool coating, the highest color variation was observed after $96 \mathrm{~h}$ of UV-B exposure. Probably, the high-energy radiation was able to modify the very external part of the coatings in a short time, thus promoting color changes. Furthermore, the $3 \mathrm{wt} . \%$ and $5 \mathrm{wt} . \%$ black cool coatings exhibited the highest color variation after $480 \mathrm{~h}$ of UV-B exposure. A color change greater than 1-2 color points could be detected by the naked eye. On the contrary, the conventional black sample achieved stable color properties, and only a small alteration of color was recorded after $480 \mathrm{~h}$ of UV-B, probably due to the presence of carbon black filler. In addition, considering the color stability of the conventional coatings, it could be deemed that the color alteration for the cool coatings was within an acceptable range. Higher stability than conventional coatings was observed in the case of all yellow cool coating samples. The change of color was related to the modification of the surface caused by UV-B irradiation. Figure 10 shows the surface of the $3 \mathrm{wt} . \%$ black sample at different UV-B exposure time observed by SEM (BED—backscattering modality). 


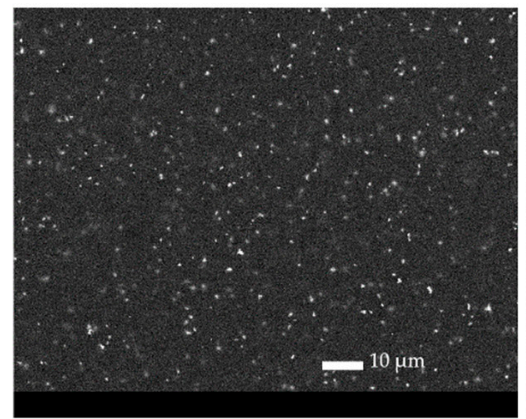

(a)

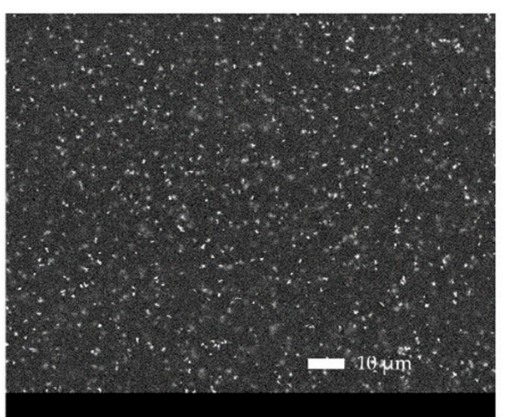

(b)

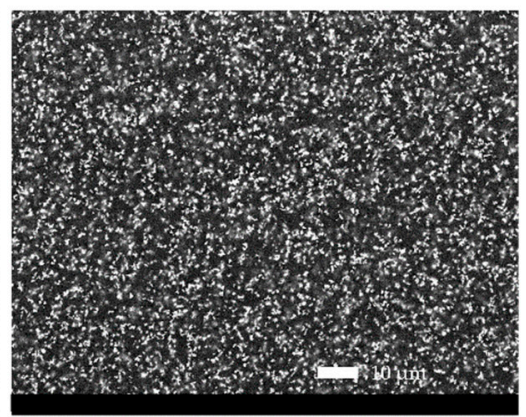

(c)

Figure 10. SEM (BED) pictures of the sample $3 \mathrm{wt} . \%$ at time 0 (a) and after $96 \mathrm{~h}(\mathbf{b})$ and $288 \mathrm{~h}(\mathbf{c})$ of UV-B exposure.

Considering the evolution of the surface appearance with exposure time (Figure 10), an increase in pigment agglomerated was observed, probably due to the deterioration of the polymeric matrix. No microcracks or other defects were present.

FT-IR analyses were collected at different exposure times in order to get information on the chemical changes produced by UV-B exposure.

Figure 11 shows the FT-IR spectra of each cool coating samples corresponding to 1 and $5 \mathrm{wt}$ \% pigment loads. The different spectra were collected at three different UV-B exposure times $(0,96$ and $480 \mathrm{~h})$.

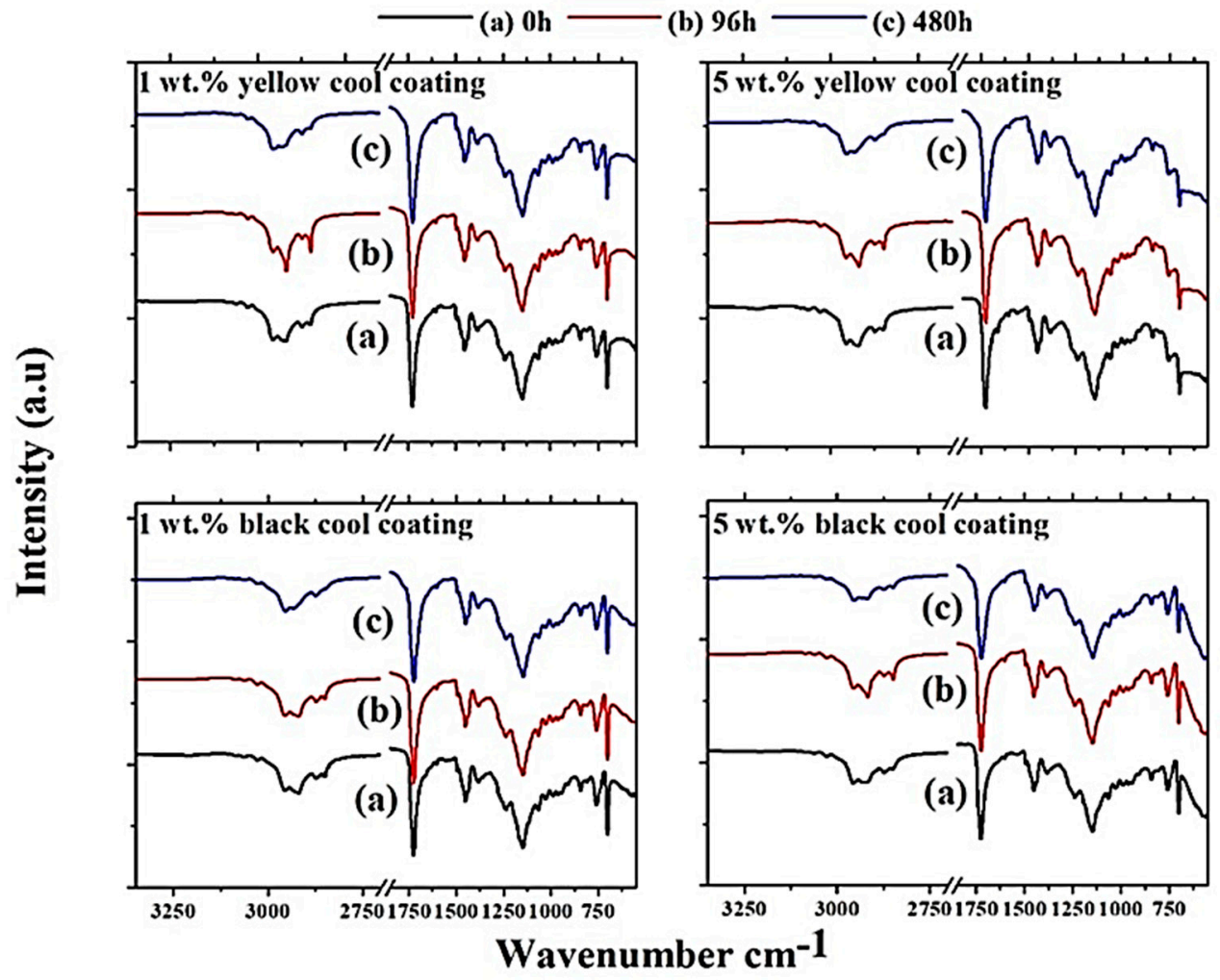

Figure 11. FT-IR spectra for each cool coating containing 1 and $5 \mathrm{wt}$ \% pigments, respectively.

The peaks observed between 2961 and $2762 \mathrm{~cm}^{-1}$ were due to $\mathrm{C}-\mathrm{H}$ aliphatic stretching of the copolymer [21]. The peak at $1725 \mathrm{~cm}^{-1}$ corresponds due to the stretching bond of $\mathrm{C}=\mathrm{O}$. The small peak observed at $1601 \mathrm{~cm}^{-1}$ was assigned to $C=C$ stretching, while the narrow peak at $1152 \mathrm{~cm}^{-1}$ was determined to be due to the vibration of $\mathrm{C}-\mathrm{O}-\mathrm{C}$ bonds [21]. The strong peak observed at 
$1152 \mathrm{~cm}^{-1}$ correlated to $\mathrm{C}-\mathrm{C} / \mathrm{C}-\mathrm{O}$ bond stretching. The peak observed at $1460 \mathrm{~cm}^{-1}$ was assigned to $\mathrm{C}-\mathrm{H}_{2}$ bending, and lastly, the peak observed at $761 \mathrm{~cm}^{-1}$ was believed to be due to $\mathrm{C}-\mathrm{H}$ rock bending [21]. The characteristic peaks were similar for each spectrum, which indicated that only small traces of chemical degradation of the coatings due to exposure time to UV-B radiation were analyzed, regardless of the pigment type/load. No reduction or acceleration of degradation was observed as a function of pigment load, as all the observed peaks had a very similar shape. The peak at $1725 \mathrm{~cm}^{-1}$ tended to broaden with exposure time to UV-B radiation [20]. This fact suggests that a photooxidative reaction between the irradiated light and the carbonyl group in the acrylic polymer occurs, which resulted in the formation of new carbonyl groups such as ketone alcohols and carboxylic acid. Furthermore, the progressive reduction of stretching aliphatic $\mathrm{C}-\mathrm{H}$ peaks at $2957-2762 \mathrm{~cm}^{-1}$ indicated that UV-B exposure tended to reduce the molecular weight of the binder material as a function of photooxidative reactions [20]. The remaining observed peaks show only minor changes and were thus not further analyzed.

The comparison of the thermal efficiency (Figure 5) with the FT-IR spectra for each type and load of pigment suggests that the observed photooxidative degradation of the coatings did not influence the thermal efficiency significantly. However, considering very small alteration in FT-IR spectra as a function of UV-B radiation exposure time and pigment load together with small variation in thermal efficiency, it was difficult to draw any straight conclusions about the influence of the binding material degradation on the optical properties of the pigments.

\section{Conclusions}

In this work, two different cool coatings were deposited by mixing complex NIR-reflective inorganic pigments with an acrylic copolymer based binding material. The study presents an accessible lab-scale approach to analyze the performance of cool coatings. This aspect has a great relevance from the industrial point of view, as the thermal behavior of a coating can be assessed with a very simple and low-cost method without the need for expensive equipment. This method of analysis could be used to evaluate the thermal performance of other types of paint systems (the type of paint and method of application). In comparison with the actual methods present in the literature, the thermal behavior evaluation allows us to get interesting results in a very simple way and with very limited cost. A possible improvement of the setup could be represented by the change of the light source (e.g., selecting the most similar to the solar radiation spectrum). Furthermore, the thermal behavior could also be investigated outdoor (with natural solar radiation) in order to have further confirmation of the validity of the method used in the laboratory scale. In addition, the influence of surface fouling on the thermal behavior of cool coatings could be very interesting. Further development could be to study the influence on the thermal behavior of the presence of openings in the walls of the scale simulacrum, representing the windows and doors.

Considering the comparison with the conventional system, the addition of black NIR pigment was more effective than that of the yellow one in limiting the warming up. While the presence of $1 \mathrm{wt} . \%$ of black pigment already seemed to be effective in reducing the temperature compared to the conventional system, the yellow pigment improved the behavior of the system only with high percentages of NIR pigment.

The highest temperature difference with the conventional coating $\left(12{ }^{\circ} \mathrm{C}\right)$ was achieved for black cool coatings. The yellow cool coating did not show a great temperature difference compared to the conventional coating.

The color characteristics of the yellow cool coating sample remained similar after $480 \mathrm{~h}$ of UV-B exposure. With the exception of the black samples with 3 and $5 \mathrm{wt} . \%$ pigments, all NIR pigmented coatings remained below two points of color delta. Instead, a decrease in gloss values was measured. Thus, the UV-B radiation damage did not produce perceptive appearance change, in particular for the samples containing the yellow pigment. In addition, the comparison of the color stability of roof and conventional coatings evidenced a color change and gloss decay of the cool coatings within an 
acceptable range. However, for some samples, in particular with 3 and $5 \mathrm{wt} . \%$ black pigments, the color change was also observed by the naked eye.

Considering the thermal behavior as a function of UV exposure, the black coatings, both the sample containing the NIR pigments and the conventional one did not show significant differences. Differently, the yellow samples showed a slight deterioration, considering the internal temperature. However, the results from the thermal assessment pointed out that coating degradation produced by $480 \mathrm{~h}$ UV-B exposure did not significantly affect the cooling properties of the cool coatings.

Considering the studied pigment types, further developments could be focused on the study of other NIR different-colored pigments without toxicity and with low environmental impact. Many pigments can be obtained in the laboratory, and their color can be changed by heat treatment.

Author Contributions: Conceptualization, S.R. and M.F.; methodology, H.L., M.F., and S.R.; formal analysis, H.L.; data curation, H.L.; writing-original draft preparation, H.L.; writing-review and editing, H.L., M.F., and S.R.; supervision, M.F. and S.R.; project administration, S.R. All authors have read and agreed to the published version of the manuscript.

Funding: This research received no external funding.

Acknowledgments: The authors would like to express gratitude towards Ferro Company (Almassora, Spain) for providing the complex NIR inorganic pigments used in this project.

Conflicts of Interest: The authors declare no conflict of interest.

\section{References}

1. Zhang, J.; Wu, L. Influence of human population movements on urban climate of Beijing during the Chinese New Year, holiday. Sci. Rep. 2017, 7, 45813. [CrossRef]

2. Konstantinidou, C.A.; Lang, W.; Papadopoulos, A.M.; Santamouris, M. Life cycle and life cycle cost implications of integrated phase change materials in office buildings. Int. J. Energy Res. 2019, 43, 150-166. [CrossRef]

3. Mavrakou, T.; Polydoros, A.; Cartalis, C.; Santamouris, M. Recognition of Thermal Hot and Cold Spots in Urban Areas in Support of Mitigation Plans to Counteract Overheating: Application for Athens. Climate 2018, 6, 16. [CrossRef]

4. Taha, H.; Sailor, D.; Akbari, H. High-Albedo Materials for Reducing Building Cooling Energy Use; UC530 31721; Lawrence Berkeley Lab: Berkeley, CA, USA, 1992.

5. Gunawardena, K.; Wells, M.; Kershaw, T. Utilizing green and blue space to mitigate urban heat island intensity. Sci. Total Environ. 2017, 584-585, 1040-1055. [CrossRef] [PubMed]

6. Zhang, L.; Deng, Z.; Liang, L.; Zhang, Y.; Meng, Q.; Wang, J.; Santamouris, M. Thermal behavior of a vertical green facade and its impact on the indoor and outdoor thermal environment. Energy Build. 2019, 204, 109502. [CrossRef]

7. Yan, L.; Qian, F.; Song, D.X.; Zheng, K.J. Research on urban heat-island effect. Procedia Eng. 2016, 169, 11-18.

8. Synnefa, A.; Santamouris, M.; Apostolakis, K. On the development, optical properties and thermal performance of cool colored coatings for the urban environment. Sol. Energy 2007, 81, 488-497. [CrossRef]

9. Cardelino, C.A.; Chameides, W.L. Natural hydrocarbons, urbanization, and urban ozone. J. Geophys. Res. 1990, 95, 13971-13979. [CrossRef]

10. Meenakshi, P.; Selvaraj, M. Bismuth titanate as an infrared reflective pigment for cool roof coating. Sol. Energy Mater. Sol. C 2018, 174, 530-537. [CrossRef]

11. Yang, R.; Han, A.; Ye, M.; Chen, X.; Yuan, L. Synthesis, characterization and thermal performance of $\mathrm{Fe} / \mathrm{N}$ co-doped $\mathrm{MgTiO}_{3}$ as a novel high near-infrared reflective pigment. Sol. Energy Mater. Sol. C 2017, 160, 307-318. [CrossRef]

12. Sameera, S.; Prabhakar Rao, P.; Divya, S.; Raj, A.K.V. High IR reflecting $\mathrm{BiVO}_{4}-\mathrm{CaMoO}_{4}$ based yellow pigments for cool roof applications. Energy Build. 2017, 154, 491-498. [CrossRef]

13. Sleiman, M. Soiling of building envelope surfaces and its effect on solar reflectance - Part II: Development of an accelerated ageing method for roofing materials. Sol. Energy Mater. Sol. C 2014, 122, 271-281. [CrossRef]

14. Yang, J.; Kumar, D.I.M.; Pyrgou, A.; Chong, A.; Santamouris, M.; Kolokotsa, D.; Lee, S.E. Green and cool roofs' urban heat island mitigation potential in tropical climate. Sol. Energy 2018, 173, 597-609. [CrossRef] 
15. Garshasbi, S.; Santamouris, M. Using advanced thermochromic technologies in the built environment: Recent development and potential to decrease the energy consumption and fight urban overheating. Sol. Energy Mater. Sol. C 2019, 91, 21-32. [CrossRef]

16. Anand, P.; Sekhar, C.; Cheong, D.; Santamouris, M.; Kondepudi, S. Occupancy-based zone-level VAV system control implications on thermal comfort, ventilation, indoor air quality and building energy efficiency. Energy Build. 2019, 204, 109473. [CrossRef]

17. Kolokotsa, D.D.; Giannariakis, G.; Gobakisa, K.; Giannarakis, G.; Synnefa, A.; Santamouris, M. Cool roofs and cool pavements application in Acharnes, Greece. Sustain. Cities Soc. 2018, 37, 466-474. [CrossRef]

18. Ferrari, C.; Touchaei, A.G.; Sleiman, M.; Libbra, A.; Muscio, A.; Siligardi, C.; Akbari, H. Effect of aging processes on solar reflectivity of clay roof tiles. Adv. Build. Energy Res. 2014, 8, 28-40. [CrossRef]

19. Thongkanluang, T.; Chirakanphaisarn, N.; Limsuwan, P. Preparation of NIR Reflective Brown Pigment. Procedia Eng. 2012, 32, 895-901. [CrossRef]

20. Yang, R.; Han, A.; Ye, M.; Chen, X.; Yuan, L. The influence of Mn/N-codoping on the thermal performance of $\mathrm{ZnAl}_{2} \mathrm{O}_{4}$ as high near-infrared reflective inorganic pigment. J. Alloys Compd. 2017, 696, 1329-1341. [CrossRef]

21. Ecco, L.; Rossi, S.; Fedel, M.; Deflorian, F. Color variation of electrophoretic styrene-acrylic paints under field and accelerated ultraviolet exposure. Mater. Des. 2017, 116, 554-564. [CrossRef]

22. Gangulya, A.; Chowdhurya, D.; Neogi, S. Performance of Building Roofs on Energy Efficiency-A Review. Energy Procedia 2016, 90, 200-208. [CrossRef]

23. Uemoto, K.L.; Sato, N.M.; John, V.M. Estimating thermal performance of cool colored paints. Energy Build. 2010, 42, 17-22. [CrossRef]

24. Driel, B.V.; Kooyman, P.; Berg, K.V.; Schmidt-Ott, A.; Dik, J. A quick assessment of the photocatalytic activity of $\mathrm{TiO}_{2}$ pigments-From lab to conservation studio. Microchem. J. 2016, 126, 162-171. [CrossRef]

25. Rossi, S.; Calovi, M.; Dalpiaz, D.; Fedel, M. The influence of NIR pigments on coil coatings' thermal behaviors. Coatings 2020, 10, 514. [CrossRef]

26. Liu, L.; Han, A.; Ye, M.; Feng, W. The evaluation of thermal performance of cool coatingscolored with high near-infrared reflective nano-brown inorganic pigments: Magnesium doped $\mathrm{ZnFe}_{2} \mathrm{O}_{4}$ compounds. Sol. Energy 2015, 113, 48-56. [CrossRef]

27. Tang, C.C.; Chen, H.I.; Brimblecombe, P.; Lee, C.L. Morphology and chemical properties of polypropylene pellets degraded in simulated terrestrial and marine environments. Mar. Pollut. Bull. 2019, 149, 11062. [CrossRef]

28. Ramdé, T.; Ecco, L.G.; Rossi, S. Visual appearance durability as function of natural and accelerated ageing of electrophoretic styrene-acrylic coatings: Influence of yellow pigment concentration. Prog. Org. Coat. 2017, 103, 23-32. [CrossRef]

29. Vedrtnam, A.; Kumar, S.; Chaturvedi, S. Experimental study on mechanical behavior, biodegradability, and resistance to natural weathering and ultraviolet radiation of wood-plastic composites. Compos. Part B Eng. 2019, 176, 107282. [CrossRef]

30. Chan-Koyoc, M.C.; Cruz-Estrada, R.H.; Cruz-Delgado, V.J.; Carrillo, J.G. Effect of Natural and Accelerated Aging on the Mechanical Performance of a Composite Based on Recycled Multilayer Carton. J. Polym. Environ. 2019, 27, 2509-2522. [CrossRef]

31. Dintcheva, N.T.; Gennaro, D.; Teresi, R.; Baiamonte, M. Pro-degradant activity of naturally occurring compounds on polyethylene in accelerate weathering conditions. Materials 2019, 12, 195. [CrossRef]

32. Tang, C.C.; Chen, H.I.; Brimblecombe, P.; Lee, C.L. Textural, surface and chemical properties of polyvinyl chloride particles degraded in a simulated environment. Mar. Pollut. Bull. 2018, 133, 392-401. [CrossRef] [PubMed]

33. Gheno, G.; Badetti, E.; Brunelli, A.; Ganzerla, R.; Marcomini, A. Consolidation of Vicenza, Arenaria and Istria stones: A comparison between nano-based products and acrylate derivatives J. Cult. Herit. 2018, 32, 44-52. [CrossRef]

34. Nguyen, T.V.; Dao, P.H.; Duong, K.L.; Duong, Q.H.; Vu, Q.T.; Nguyen, A.H.; Mac, V.P.; Le, T.L. Effect of $\mathrm{R}_{-} \mathrm{TiO}_{2}$ and $\mathrm{ZnO}$ nanoparticles on the UV-shielding efficiency of water-borne acrylic coating. Prog. Org. Coat. 2017, 110, 114-121. [CrossRef]

35. Carmona-Quiroga, P.M.; Jacobs, R.M.J.; Viles, H.A. Weathering of two anti-graffiti protective coatings on concrete paving slabs. Coatings 2017, 7, 1. [CrossRef]

36. Gheno, G.; Ganzerla, R.; Bortoluzzi, M.; Paganica, R. Accelerated weathering degradation behaviour of polyester thermosetting powder coatings. Prog. Org. Coat. 2016, 101, 90-99. [CrossRef] 
37. Hamaviriyapornwattana, N.; Sombatsompop, N.; Markpin, T.; Kositchaiyong, A.; Wimolmala, E. Solar reflectance, surface adhesion, and thermal conductivity of wood/natural rubber composite sheet with $\mathrm{TiO}_{2} /$ polyurethane topcoat for roofing applications. J. Vinyl. Addit. Technol. 2012, 18, 184-191. [CrossRef]

38. Hu, J.; Gao, J.; Li, X.; Du, C. An investigation of UV photo-degradation on acrylic polyurethane varnish coatings. J. Chin. Soc. Corros. Prot. 2009, 29, 371-375.

39. Hu, J.W.; Li, X.G.; Gao, J. Electrochemical evaluation of UV aged acrylic polyurethane varnish coatings. Corros. Protect. 2009, 30, 211-214.

40. Liu, M.; Horrocks, A.R. Effect of carbon black on UV stability of LLDPE films under artificial weathering conditions. Polym. Degrad. Stabil. 2002, 75, 485-499. [CrossRef]

41. Scrinzi, E.; Rossi, S.; Deforian, F. Influence of natural and artificial weathering on aesthetic and protective properties of organic coatings. Corros. Rev. 2011, 29, 275-285. [CrossRef]

42. Rossi, S.; Fedel, M.; Petrolli, S.; Deflorian, F. Accelerated weathering and chemical resistance of polyurethane powder coatings. J. Coat. Technol. Res. 2016, 13, 427-437. [CrossRef]

43. Cambier, S.M.; Frankel, G.S. Filiform corrosion of polyvinyl butyral-and bisphenol a-based epoxy-coated steel after standard laboratory exposures. Corrosion 2014, 70, 1230-1237. [CrossRef]

44. ASTM G154-06. Standard Practice for Operating Fluorescent Light Apparatus for UV Exposure of Nonmetallic Materials; ASTM International: West Conshohocken, PA, USA, 2006.

45. Bendiganavale, A.; Malshe, V. Infrared Reflective Inorganic Pigments. Recent Pat. Chem. Eng. 2008, 1, 167-179.

46. Technical Datasheet: Ferro Pigments for Coatings. Available online: www.ferro.com/-/media/files/resources/ pigments-and-dispersions/technical/ferro-pigments-for-coatings-2018-emea.pdf (accessed on 10 August 2020).

47. Levinson, R.; Berdahl, P.; Akbari, H. Solar spectral optical properties of pigments-Part II: Survey of common colorants. Sol. Energ. Mater. Sol. C 2005, 89, 351-389. [CrossRef]

48. Hård, A.; Sivik, L.; Tonnquist, G. NCS Natural Color System-From Concepts to Research and Applications. Part I and II. Color Res. Appl. 1996, 21, 180-220. [CrossRef]

49. Carter, E.C.; Ohno, Y.; Pointer, M.R.; Robertson, A.R.; Seve, R.; Schanda, J.D.; Witt, K. Colorimetry, CIE 15: Technical Report, 3rd ed.; Commission Internationale de L'éclairage: Vienna, Austria, 2004.

50. Zubielewicz, M.; Kaminska-Tarnawska, E.; Slusarczyk, A.; Langer, E. Prediction of heat build-up of solar reflecting coatings based on physico-chemical properties of complex inorganic colour pigments (CICPs). Prog. Org. Coat. 2011, 72, 65-72. [CrossRef]

51. Radhi, H.; Assem, E.; Sharples, S. On the colours and properties of building surface materials to mitigate urban heat islands in highly productive solar regions. Build. Environ. 2014, 72, 162-172. [CrossRef]

Publisher's Note: MDPI stays neutral with regard to jurisdictional claims in published maps and institutional affiliations.

(C) 2020 by the authors. Licensee MDPI, Basel, Switzerland. This article is an open access article distributed under the terms and conditions of the Creative Commons Attribution (CC BY) license (http://creativecommons.org/licenses/by/4.0/). 